\title{
Sciendo
}

\section{Context-sensitivity and (Indicative) Conditionals}

\author{
Pedro Santos \\ Universidade do Algarve \\ Universidade de Lisboa
}

Disputatio Vol. 2, No. 24

May 2008

DOI: $10.2478 /$ disp-2008-0003

ISSN: 0873-626X 


\title{
Context-sensitivity and (indicative) conditionals
}

\author{
Pedro Santos ${ }^{1}$ \\ University of the Algarve and \\ The Philosophy Centre, University of Lisbon
}

\begin{abstract}
Conditionals (in particular indicatives) give rise to stand-offs that have become well known from Gibbard's initial Sly Pete example. The stand-offs can be seen as evidence for the context-sensitivity of (indicative) conditionals and arguably do not involve disagreement. I claim that the latter feature lends credibility to an indexical treatment of indicatives.
\end{abstract}

\section{Conditionals in stand-off environments}

It has been pointed out that indicative conditionals give rise to standoffs along the following lines. A conditional of the form (a) If $A$ then $C$ may be judged as true ${ }^{2}$, by a certain speaker, for her own communicative purposes, in circumstance of evaluation $e$, while at the same time a conditional of the form (b) If $A$ then not- $C$ may also be judged as true by another speaker in an equally legitimate way, in $e$. Assuming that no one would simultaneously condone two conditionals with the same antecedent and contradictory consequents, this means that the two speakers would have contradictory responses to each conditional. In particular, if someone accepts (a) she will deny (b) in the same context of utterance. Furthermore, no third party would have a reason to choose between the two conditionals, again because each of them seems to be asserted on equally legitimate grounds. This feature of indicative conditionals is illustrated by the well-known Sly Pete example (originally Gibbard's ${ }^{3}$ ) of which I now give an abridged

${ }^{1}$ Work partially supported by the project Content, POCI/FIL/55562/2004 (FCT) at the Philosophy Centre of the University of Lisbon.

${ }^{2}$ For the purposes of this paper, I assume that conditionals have truth-values (a view that is not popular with a few authors, notably D. Edgington and Gibbard).

${ }^{3}$ Cf. Gibbard 1981: 226 ff.

Disputatio, Vol. II, No. 24, May 2008 
version. Sly Pete is playing poker and has to decide whether to call or fold. Two people, $\mathrm{X}$ and $\mathrm{Y}$, are also present. $\mathrm{X}$ knows that Pete is cheating and saw his opponent's hand. Y knows that Pete's hand is weaker than his opponent's. $\mathrm{X}$ and Y do not share each other's knowledge; and both leave the room before Pete makes up his mind. Under these circumstances, X's evidence is enough to support the assertion of the following indicative:

(1) If Sly Pete called, he won.

since he knew his opponent's hand, and would therefore have refrained from calling unless he knew he was going to win.

On the other hand, Y's evidence is enough to support the assertion of the following indicative:

(2) If Sly Pete called, he lost.

since he had the weaker hand.

The phenomenon is quite widespread and the examples are numerous. The following two differ slightly from the Sly Pete case.

One is due to Edgington ${ }^{4}$. Three people, A, B and C are known to be the only mobsters in a room with a Mafia boss. X and Y, two FBI agents, are spying on them from different angles. $\mathrm{X}$ sees $\mathrm{C}$ leave, after which he hears the boss giving some orders. She then confidently asserts

(3) If A didn't receive the orders, B did.

Y, on the other hand, sees B leave before the orders are given. She then confidently asserts

(4) If A didn't receive the orders, $\mathrm{C}$ did.

There is, again, little point in trying to decide which one of the conditionals is better grounded on facts (the obvious fact that it was A who received the orders does not adjudicate between them). Rather, the assertion of both conditionals seems warranted because different criteria are being used for selecting the relevant antecedentcircumstances is being used. A similar example is presented by Ben-

${ }^{4}$ Edgington 2003: 23. 
nett $^{5}$. In a dam, a main channel (controlled by Top Gate) bifurcates into two sub-channels going east and west. These, in turn, are controlled by East Gate and West Gate respectively. If East Gate is opened and Top Gate opens, all the water will run eastwards; if West Gate is opened and Top Gate opens, all the water will run westwards. If both the east and west gates are open nothing happens as Top Gate will then be closed - the irrigation system does not allow for the three gates to be open at the same time. Observer X sees the East Gate open and is therefore vindicated in asserting

(5) If Top Gate opens, all the water will run eastwards.

Whereas Y sees the West Gate opened and is therefore vindicated in asserting

(6) If Top Gate opens, all the water will run westwards.

Once more, it makes no sense to say that one of the observers is right while the other is wrong; the fact that Top Gate will not open does not weaken either statement. Once more, it makes no sense to claim that one of the conditionals is better grounded on facts than the other.

In this kind of completely symmetric stand-off cases, then, both conditionals are equally warranted. There clearly is no justification for choosing one of the conditionals as the 'right' one to assert. In the mobster example, the fact that mobster B left the room and the fact that mobster $\mathrm{C}$ left the room provide the same sort of evidence for the assertion of a conditional. Therefore, if the assertion of (5) is warranted by the evidence available to $\mathrm{X}$, so must the assertion of (6) be warranted by the evidence available to $\mathrm{Y}$. And the same goes for the dam example.

What about the non-symmetric cases illustrated by $(1) /(2)$ ? It could perhaps be argued that in each of those cases the evidence warranting the assertion of the conditionals is different in nature, since on the one hand we have Pete's cheating and the expected consequences of that fact concerning his decision to call or fold, and on the other hand we are faced with the hard fact that he has the weaker hand. But this is wrong, I think. On the face of it there is no reason why a criterion for endorsing a conditional that is based on

\footnotetext{
${ }^{5}$ Bennett 2003: 85 .
} 
facts about who has the weaker hand should count as weaker or less capable of supporting the assertion of a conditional than one which is based on facts about Pete's cheating. One could say that a disposition to avoid calling if one knows one has the weaker hand is nothing more than, well, a disposition - one which can be defeated if the occasion arises. But the point being made by $\mathrm{X}$ in asserting (1) is precisely that Sly Pete has that disposition and her assertion is vindicated to the extent that he does.

Let me now list a few central features of the kind of stand-offs I have been discussing:

(a) The two conditionals can jointly be used to show that $\mathrm{A}$ is false, assuming that both kinds of evidence are trustworthy ${ }^{6}$.

(b) The two conditionals are jointly unassertible, despite the fact that they are jointly assertible by two speakers who assert the conditionals from different stances, taking as relevant different features of one and the same situation.

(c) Both $\mathrm{X}$ and $\mathrm{Y}$ have strong evidence for their respective conditionals; neither can be said to be making a mistake in uttering it; each is making a cogent point. So, despite b), there is little room for claiming that one of the conditionals must be false. There just seems to be no context-independent criterion establishing whether the consequent is true if the antecedent is true, thus falsifying one of the conditionals.

These remarks go counter what Lycan calls the Hard Line on conditional stand-offs (henceforth, HL). By and large, one is a hardliner on conditional stand-offs if one makes use of the Principle of Conditional Non-Contradiction, i.e.

(CNC) Two conditionals of the form If $A$ then $B$ / If $A$ then not- $B$ cannot both be true (at the same circumstance of evaluation).

to claim that that conditionals giving rise to stand-off situations cannot both be true. Could HL be right, in which case the above remarks are essentially inaccurate?

On the face of it, one might feel inclined to say that HL provides an attractive account of the semantics of conditionals (as opposed to their pragmatics), given the intuitive cogency of CNC. In his book

\footnotetext{
${ }^{6}$ Cf. Edgington 1995: 278.
} 
Real Conditionals, nevertheless, Lycan argues it cannot be right. I think Lycan is fundamentally right on this point.

What exactly could be the case for HL? A hardliner about indicatives would play down the role of contextual idiosyncrasies (e.g. the speaker's specific and partial stance) in legitimising the acceptance of, say, (1) and (2) and therefore its relevance as a criterion for assessing an indicative's truth value. The idea underlining this position is, in other words, that contextual factors and in particular the communicative intentions of speakers cannot determine what truth-value a given conditional may have (provided, of course, any indexical items it contains have been assigned their referents), since those factors impinge neither on what the semantic content of a conditional is nor on the relevant features of the circumstance of evaluation at which it is being assigned a truth-value. For a hardliner, $\mathrm{X}$ is ignorant of a crucial fact about the poker game, namely that Sly Pete has the weaker hand; if she were aware of that fact, she would retract her conditional and agree with $\mathrm{Y}$ that (2) expresses the correct view on what the outcome of the game was if Sly Pete called. So the assertion of (1) is somewhat justified within the confines of X's limited knowledge, but the conditional is itself false, since $\mathrm{X}$ failed to take into account all the relevant facts. In a Sly Pete kind of situation, the hardliner contends, hard facts like Pete's having the weaker hand prevail over beliefs derived from expected consequences of, say, Pete's cheating. In other words, when we are trying to establish whether Pete won if he called, it seems that the bottom line lies in whether Pete's hand allowed him to win. Therefore, since Sly Pete has the weaker hand, (1) cannot be described as true.

I agree with Lycan that this is not a correct verdict on Sly Pete stand-offs. It just doesn't seem right to dismiss (1) as false although partially justified given the lack of evidence available to the speaker. Lycan points out that none of the typical features of the justified assertion of false sentences is present in a standard stand-off situation. The assertion of (1) by X isn't supported by a false belief; nor is X relying on some kind of misleading evidence such as someone would be if, say, they relied on the fact that John owned a car on Friday evening to conclude that he still owned it on Saturday at noon, thus overlooking the fact that he actually sold it to a friend on the first hours of Saturday morning; nor is the assertion of (1) supported by an inductive piece of reasoning; rather, $\mathrm{X}$ seems to be relying on the following deductive argument: 
(1') Pete didn't call unless he knew he had the strongest hand. If Pete called and he had the strongest hand, then he won. Therefore, if Pete called, he won.

Still, it may be argued that some asymmetry exists between the two conditionals in stand-off situations like those illustrated by (1)/(2). Lycan concedes, in particular, that in some cases one of the conditionals can be retracted when the person who's asserted it becomes aware of the fact which was crucial in warranting the other speaker's assertion of her conditional. For instance, $\mathrm{X}$ is bound to retract her assertion of (1) when she finds out that Sly Pete had in fact the weaker hand. It should be noted that the converse retracting cannot reasonably be envisaged: Y would find it difficult to retract her assertion of (2) if she found out that Sly Pete was cheating. So there does seem to be some asymmetry between the two conditionals in at least some stand-off situations, which might vindicate the thesis that one of them is true while the other is false (again, presuming one adopts the view that indicatives have truth-values). But this conclusion would be unwarranted, I think. In those cases where the retracting does occur, it is not the case that the retracting agent/speaker acknowledges being wrong before; in fact, she typically would stick to her previous point, and would be warranted in doing so. It is just that she must now express it by means of the counterfactual version of her initial conditional, since she is now aware that the antecedent is false (for instance, $\mathrm{X}$ becomes aware that Sly Pete did not call, since he must have known he had the weaker hand). By asserting that counterfactual, i.e.

(7) If Sly Pete had called, he would have won.

she will be making the same point as she would originally have been making and, one might add, she will be literally saying much same thing as before. So HL does not seem to deal very well with asymmetric stand-offs. And, quite obviously, it has little to say about symmetric ones. Overall, then, HL does not seem to hold for indicatives.

\section{Stand-offs, the Hard Line and counterfactuals}

Frank Jackson (in Jackson (1990)) used the stand-offs to argue for a view I will call the Apartheid view on counterfactuals and indicatives, i.e. the theory according to which there is the semantic or truth- 
conditional divide between both kinds of conditional. His argument, in abridged version, is as follows. The existence of stand-offs shows that any pair of indicatives of the form If $A$ then $B$ / If $A$ then not- $B$ are mutually consistent, contrary to what is predicted by Conditional non-Contradiction (CNC). In other words, Jackson claims that $\mathrm{CNC}$ does not apply to indicatives such as (1)/(2). On the other hand, Jackson also claims, any two counterfactuals of the same form (i.e. with the same antecedent and contradictory consequents) are mutually inconsistent, therefore complying with $\mathrm{CNC}$, as shown when one considers pairs of counterfactuals being evaluated in similar settings as the indicatives (1)/(2). Thus the argument is intended to establish a difference in logical behaviour between counterfactuals and indicatives; and so, on the grounds that logical behaviour supervenes on truth-conditions, it purports to establish Apartheid.

What Jackson is saying, in other words, is that HL holds for counterfactuals. He claims that there are no real stand-offs in the counterfactual case, because when we translate the conditional pairs discussed above into counterfactual form (which basically means into the subjunctive $\operatorname{mood}^{7}$ ), we get a true one and a false one, which shows that counterfactuals, contrary to indicatives, comply with CNC. Jackson is therefore claiming that compliance with $\mathrm{CNC}$ is inconsistent with the ability to generate genuine stand-offs.

I think Jackson is wrong on both counts — in adopting HL for counterfactuals and in equating compliance with CNC with the impossibility of stand-offs. Assuming some version of the LewisStalnaker possible-world semantics for counterfactuals, whether or not a counterfactual is true in a world depends, roughly, on whether the closest antecedent-worlds are also consequent-worlds. If we construe 'closer' as 'more similar', it depends on whether the similarity relation on the set $\mathrm{W}$ of accessible worlds yields a set $\mathrm{S}$ of antecedent-worlds (i.e. worlds where the antecedent holds) in which the consequent is true such that all members of $\mathrm{S}$ are more similar to the actual world than any antecedent-world in which the consequent is false. Jackson would be right if there were a unique such similarity measure between worlds. But, as a closer look at the counterfactual

\footnotetext{
${ }^{7}$ I will not be discussing here the identification of counterfactuals with subjunctives; it is widely acknowledged, at least, that not all subjunctives are counterfactuals (cf. Anderson's 'if Jones had taken arsenic, he would show the symptoms he is actually showing', normally used to argue for the thesis that Jones did take arsenic).
} 
versions of Sly Pete cases show, there is no such unique measure, a point convincingly made in Lowe (1991). In a counterfactual Sly Pete sort of case (i.e. a situation where $\mathrm{X}$ asserts (7) and $\mathrm{Y}$ its contrary 'If Sly Pete had called, he would have lost'), we are faced with two different measures of world similarity. On the one hand, from X's point of view, the most similar antecedent-worlds must be ones where Sly Pete is cheating (and is, presumably, intent on winning). On the other hand, from Y's stance, the most similar antecedentworlds must be ones where Sly Pete has the weaker hand. It is pretty clear that one must choose between these two criteria for selecting the most similar antecedent-worlds: an antecedent-world where an hybrid criterion one was used (i.e. one according to which most similar antecedent-worlds are ones where Sly Pete was cheating, had the weaker hand and yet called) is not similar enough to be worth taking into account. Furthermore, as in the indicative case, there is little room for claiming that one of the two criteria prevails over the other $^{8}$. So it seems that the two different ways of selecting a set of closest antecedent-worlds (and the corresponding contradictory verdicts they yield about whether only worlds where Pete won, respectively lost, are in that set) are equally legitimate; neither can be described as a more apt implementation of the notion of 'most similar antecedent-worlds.' We can conclude that counterfactuals do allow for (genuine) stand-offs, contrary to what Jackson claims. Arguably, then, HL does not apply to the counterfactual case any more than it does to the indicative case. ${ }^{9}$

In fact, hardliners of either variety tend to overlook the contextsensitivity displayed by conditionals. Such context-sensitivity is, by the way, the reason why rejecting HL does not entail rejecting CNC: once we recognize that conditionals are generally content-sensitive in the way described, we are vindicated in claiming that both indicatives

\footnotetext{
${ }^{8}$ The fact that one must choose between two criteria for selecting antecedentworlds in order to avoid ending up with worlds too irrealistic to be relevant does not, by itself, entail that both criteria are equally legitimate; this result is only vindicated if neither criterion supersedes the other. But, as we have seen, that seems to be the case.

${ }^{9}$ A similar point has been made by Edgington (1997), although in connection with her bold claim that counterfactuals appearing in stand-off environments are neither true nor false, and are therefore a counter-example to the Stalnaker-Lewis analysis of counterfactuals.
} 
and counterfactuals comply with $\mathrm{CNC}$ given a context of utterance: as we have seen, in a stand-off situation the speaker endorsing or asserting If $A$ then $B$ cannot legitimately deny that conditional - for instance, by asserting If $A$ then not $B$ - unless, of course, he adopts the other contender's stance ${ }^{10}$.

One thing to notice about stand-offs is that that they illustrate a feature of conditionals (both counterfactual and indicative) that is often overlooked. In fact, if we go through all the examples discussed so far, we can see that the epistemic constrains of each context of utterance do not by themselves determine which conditional a given speaker is vindicated in asserting. X may become aware that Sly Pete has the weaker hand and still want to make the point that his cheating is inconsistent with his loosing by means of the assertion of a conditional - in this case, as we have seen, the counterfactual (7). (The same goes, of course, for symmetric stand-offs.) In some cases, not even a change of mood is called for. This is illustrated by an example Lycan discusses in connection with the stand-offs (slightly changed) ${ }^{11}$. One might say of Jones

\footnotetext{
${ }^{10}$ There is another reason why stand-offs fail to provide evidence for a semantic divide between counterfactuals and indicatives. As we have seen, it is surely the case that if X learns that Pete has the weaker hand, her assertion of (1) is no longer warranted, unlike her assertion of (7) (which would still count as true provided she chose the adequate similarity measure). But this does not have to mean either that counterfactuals and indicatives appeal to fundamentally different semantic mechanisms or that HL holds for indicatives. Rather, it can be taken to mean that there really does not seem to be much of a difference between the way counterfactuals and indicatives express conditionality, besides the commitment toward the truthvalue of the antecedent. In the Sly Pete case, since $\mathrm{X}$ is now aware that Pete has the weaker hand, she is also aware that Pete did not call, as she also knows that he is cheating. In other words, she is now aware that the antecedent of (1) is false. But, crucially, she can still be described as making the exact same point as she had been making in the original version of the example (by uttering (1)), if she utters the counterfactual version of (1), to exactly the same effect. Arguably, then, in both cases she will be making essentially the same claim, viz. that all envisageable (from her perspective) circumstances where Pete called are ones where he was cheating and therefore ones where he won. The reason why the assertion of (1) is unwarranted in X's enhanced epistemic context is not that (1) and (7) have totally different semantic contents; rather, it is simply that in the new context $\mathrm{X}$ can no longer reasonably take the truth of the antecedent as an actual possibility and therefore can no longer assert (1) felicitously.

${ }^{11}$ Lycan 2001: 196.
} 
(8) If Jones jumped out of this $6^{\text {th }}$ storey window, he got badly hurt.

Or, in the same situation and building on the same known facts, one might deny (8) an endorse (9) instead:

(9) If Jones jumped out of this $6^{\text {th }}$ storey window, he didn't get hurt.

As in other stand-off examples, one may choose to build on the fact that $6^{\text {th }}$ storey windows are too high for Jones not to get hurt when jumping out of one of them (which legitimises the assertion of (8)), or one may choose to build on the fact that Jones is the kind of guy who would not jump out of $6^{\text {th }}$ storey windows without making sure that a safety net or suchlike was available on the occasion (which legitimises the assertion of $(9))^{12}$. In this case, both speakers have the same evidence (both know what a $6^{\text {th }}$ storey window is, both know that Jones has no flying powers, and both know that he would in all likelihood have made sure the safety net was there). So it is clearly a question of choice between which point one chooses to make. Interestingly, unlike more standard stand-off situations, in this case both speakers can legitimately switch from one conditional to the other without resorting to change of mood. But these somewhat exceptional cases illustrate a general feature of stand-offs: that the choice of which conditional to assert hinges on what the communicative intentions of the speakers are; stand-offs as the one exemplified by (8)/(9) merely stress that point in a particularly vivid way. Just as in the more standard Gibbardian situations discussed above, the speaker asserting (8) can, on another occasion but supported by the same kind of background information, choose to make another point by denying that conditional and/or by asserting a conditional with the same antecedent and a contradictory consequent (i.e. by asserting (9)); but she would not thereby necessarily be retracting her previous assertion, nor would the other speaker necessarily be disagreeing with her if she stuck to asserting (8).

\footnotetext{
${ }^{12}$ In his 'Counterfactual Dependence and Time's Arrow', Nous, 13, 455-476, David Lewis identified backtracking counterfactuals If $A$ then $B$ as ones analysable as 'If event $A$ were to happen at $t_{A}$, then event $B$ would happen at $t_{B}$ ' where $t_{B}$ precedes $t_{A}$. What examples like Sly Pete, the 6th storey jump and others seem to show is that the notion has an indicative counterpart.
} 
One natural way of accounting for the behaviour of indicative conditionals (and perhaps also counterfactuals) in stand-off situations is as follows. The selection of the range of relevant antecedentcircumstances changes according to the point being made in asserting a given conditional, so that in a stand-off the set of antecedentcircumstances being stated to be consequent- ones and nonconsequent ones are different. This is why, one might add, both assertions come across as warranted and also why, provided we accept that indicatives have truth-values, it would be reasonable to describe both as true. The natural corollary of this line of reasoning is that idiosyncratic features of contexts of utterance (notably, speaker's communicative intentions) not only interfere in legitimising the assertion of the conditionals in a stand-off situation, but apparently also play a central role in determining what propositions speakers are literally expressing in uttering a given conditional.

In the Sly Pete case, someone making the point (whether counterfactually or not, by means of the utterance of either (1) or (7)) that Sly Pete was cheating is talking about hypothetical circumstances where Sly Pete called and was cheating - she is saying that in all of them, Sly Pete won; whereas someone making the point (by means of either (2) or its counterfactual counterpart) that he could not have called and won because he had the weaker hand, is talking about hypothetical circumstances where he called and had the weaker hand — she is saying that in all of them, Sly Pete lost. In a stand-off situation, then, it seems that which proposition is literally expressed by If $A$ then $C$ in a context where its assertion is warranted differs from what would be being said by that same conditional in a context where the assertion of If $A$ then not- $C$ is warranted (and therefore, in a context where the denial of If $A$ then $C$ is warranted). Ultimately, this seems to be the reason why even if we reject HL, stand-offs cannot be used to question $\mathrm{CNC}$ for either indicatives or counterfactuals. On an account of stand-offs along these lines, CNC is preserved in both cases by making room for the indexicality of conditionals (or of 'if'). Admittedly, for some semanticists, this might seem too high a price to pay for making sure a logical principle applies to a certain type of natural language construction in just the way our intuitions say it does. But those semanticists would have to show where intuitions about both the context-sensitivity of conditionals and the cogency of $\mathrm{CNC}$ have led us astray in accounting for conditional stand-offs. 


\section{Context-sensitivity and indexicality}

In this paper I have been suggesting that an indexical treatment of indicative conditionals and perhaps also of counterfactuals is the most reasonable way of tackling conditional stand-offs. More specifically, the suggestion is that, granting that conditionals display some degree of context-sensitivity (as stand-off situations arguably show), it is reasonable to see them (or, more specifically, 'if') as indexical items. As also suggested, one way of making sense of this indexicality would be to assign truth-conditions to conditionals along the following lines: the set of antecedent-worlds is being asserted to be consequentworlds depend on particular aspects of the utterance situation, notably the speaker's intended criterion for selecting antecedent-worlds.

Nevertheless, as MacFarlane ${ }^{13}$ has pointed out, cases of contextsensitivity do not have to treated as cases of indexicality. In stand-offs involving predicates of taste, or epistemic modals, or 'know' (to list just a few of the much debated examples of non-standard, nonKaplean context-sensitivity), the context-sensitivity they display is consistent with the sentences uttered being non-indexical. Take the context-sensitivity displayed by, say, statements involving predicates of (aesthetic) taste in situations where speaker A asserts a sentence $S$ and speaker B denies A's assertion (typically, by asserting its contra$\operatorname{dictory}^{14}$ ): if A asserts 'this is a great painting' and B asserts 'no, how can you say that, it's awful', it seems that both speakers' speech acts are vindicated by their respective standards of aesthetic taste; in other words, it seems that which aesthetic statement A or B are going to endorse (and so whether B is going to deny A's assertion) is determined by their favoured standard; also, apparently no adjudication from neutral ground between opposing statements is possible, so to speak - for there does not seem to be any such neutral ground ${ }^{15}$.

${ }^{13}$ In MacFarlane (forthcoming).

${ }^{14}$ Denial by the assertion of contraries seems to be somewhat idiosyncratic of conditional stand-offs.

${ }^{15}$ Of course, giving credit to MacFarlane's point on context-sensitivity does not entail agreeing with his relativistic treatment of many of the cases of contextsensitivity he discusses. In some of them such a treatment may strike us as more objectionable than in others (e.g. moral stand-offs vs gastronomical stand-offs), but that discussion is beyond the scope of this paper. 
Now, MacFarlane argues that such cases of context-sensitivity are consistent with one and the same propositional content being asserted and denied. It could be that what varies is, in Kaplan's terminology, the circumstance of evaluation in which sentences containing predicates of taste, sentences containing epistemic modals or others displaying some degree of context-sensitivity are assessed for truth. According to MacFarlane, adopting such a view on context-sensitivity will ensure that we will be able to deal with cases of genuine disagreement involving utterances of those sentences.

To make a complicated story simple, the phenomenology of disagreement can be described as involving speech acts of denial (as when someone asserts the contradictory - or contrary - of some previously asserted sentence) and retracting (as when someone retracts her own previous assertion and endorses its contradictory - or contrary - on the basis of someone else's utterance of that contradictory or contrary). It is debatable whether denial and retracting are necessary or sufficient conditions for disagreement but for the purposes of this paper I will go along with MacFarlane's claim that on an occasion where speaker A asserts 'this is a great painting' and speaker $\mathrm{B}$ asserts 'no, it's awful' $\mathrm{A}$ and $\mathrm{B}$ are in fact disagreeing ${ }^{16}$. For these cases of disagreement, MacFarlane recommends what he calls a 'nonindexical contextualist' treatment. The argument runs as follows: the kind of context-sensitivity under analysis cannot be built into propositional content, i.e. contextual elements determining a statement's truth-value (e.g. contextual idiosyncrasies determining different truth values according to particular stances, e.g. standards of taste) cannot be 'content-determinative'; for if they were, whenever someone asserted S and someone else asserted not-S (S being one of kinds of sentence under discussion) different propositions would be being affirmed and denied, as contextual idiosyncrasies concerning, say, standards of aesthetic taste would then be part of the propositional content asserted by each speaker (A's 'this is a great painting' would then be equivalent to 'this is a great painting for me', while B's 'it's awful' would be equivalent to 'it's awful for me'), which would mean that each party was making a statement about herself. This would

${ }^{16}$ The same goes for examples involving, say, epistemic modals: if A endorses 'Ann might be in Évora' and B endorses 'Ann could not be in Évora', then A and B disagree (namely, about there being enough evidence to exclude the possibility that Ann is in Évora). 
arguably preclude accounting for such exchanges in terms of disagreement. MacFarlane therefore proposes that context-sensitivity of this kind is to be construed as 'circumstance-determinative' rather than 'content-determinative. ${ }^{17}$

In this paper I will make no claims about statements involving predicates of taste, epistemic modals, 'know' or any other frequently discussed alleged cases of non-standard context-sensitivity. Perhaps MacFarlane is right them in claiming that they give rise to genuine disagreement. And perhaps he is right in claiming that a non-indexical view provides the best account for cases where genuine disagreement is present. Of course, on the assumption that MacFarlane is right, if conditionals behaved like statements of taste in that respect, it would be hard to deny them a 'non-indexical contextualist' treatment (be it MacFarlane's variety or any other), at least if we take their contextsensitivity seriously. But the context-sensitivity arguably present in conditionals (in particular, in indicatives) does not seem to follow this pattern; there seems to be no genuine disagreement going on in conditional stand-offs. Instead, as noted above, speakers asserting If $A$ then $B$ and If $A$ then not $B$ are seemingly talking past each other, rather than contradicting each other. When aware of each other's reasons for asserting the other conditional, they are bound to grant each other legitimacy in their assertions/denials and would indeed have endorsed each other's utterance if they had had access to the same kind of evidence. More importantly, they would have done so without eschewing their previous statement; so there is little room for claiming that in

${ }^{17}$ This would entail a more sophisticated conception of what a circumstance of evaluation is, of course. Another complication with MacFarlane's proposal is the following. Clearly, for a genuine case of (verbal) disagreement to arise, not only the same propositional content must be both affirmed and denied, but also the same circumstance of evaluation must be assumed to be operative for both speech acts. Otherwise, one would not be able to talk about two parties disagreeing, as each one's statement would be being assessed against different criteria (say, in different possible worlds). In MacFarlane (2007b), MacFarlane therefore proposes a specific brand of non-indexicalism, according to which in each instance of assessment, the stance that counts as relevant is the assessor's. This is argued to ensure disagreement, for in a stand-off involving, say, predicates of taste standards of accuracy for evaluating the two parties' statements are measured from the assessor's perspective and therefore only her statement is to be judged accurate; since from the other party's perspective, instead, only her own standards are to be counted as reliable, disagreement is ensured. It is debatable whether this relativistic treatment of disagreement situations is workable, but I will not be pursuing this discussion here. 
adhering to each other's viewpoint, the speakers are retracting their initial statement. It is true that in some cases (namely the nonsymmetric ones) access to new information calls for the speaker to refrain from her previous assertion of an indicative conditional (remember the speaker initially aware of Pete's cheating and later finding out about his weaker hand), but, as we have seen, in those cases she would be able to resort to its counterfactual counterpart in order to make exactly the same point as before (see note 10); so that the claim that some retracting is going on in such situations is less than convincing. This is even more obvious in symmetric cases. It is true that, also in those cases, acknowledging the other party's legitimacy in her previous assertion involves realizing that the common antecedent of both conditionals is false; it therefore also involves rephrasing one's initial indicative as a counterfactual. But, given the symmetry that characterizes these cases, there is no difference in the degree of assertibility of the two indicatives and therefore such realization could never lead to the retracting of one's previous assertion. In other words, in stand-off situations like the ones discussed above there is no evidence of genuine disagreement (as opposed to fake, or pseudo-disagreement).

Regardless of what the exact characterization of disagreement in terms of necessary and sufficient conditions might be, it is reasonable to say that identity of propositional content and identity of circumstance of evaluation are jointly sufficient conditions for disagreement in assertion/denial settings as the ones under discussion. For instance, two people making opposing statements of taste (affirming contradictory or contrary propositions or affirming and denying one and the same proposition), in a setting where one and the same standard of taste is operative, are surely in disagreement. By Modus Tollens, it follows that where there is no disagreement, as in conditional standoffs, the same propositional content is at stake in both parties' statements only if the circumstances of evaluation in which those statements are to be assessed are different (if we also had the same circumstances of evaluation, sameness of propositional content would yield disagreement). In other words, in affirming/denying environments where there is no disagreement, evidence about sameness of content strongly argues for the claim that the statements are assessed against different circumstances of evaluation and therefore for nonindexical contextualism, since the suggestion would thereby be vindicated that the relevant kind of context-sensitivity is to be construed as circumstance-determinative rather than as content-determinative. In particu- 
lar, if evidence was available for identity of content in the conditionals being asserted/denied in a stand-off situation, that would count as an argument, indeed the argument, for their context-sensitivity to be dealt with non-indexically. One would thereby be acknowledging the context-sensitivity of (indicative) conditionals without being committed to treating, say, 'if' as an indexical item.

The connection between lack of disagreement, sameness of content and non-indexicalism can be viewed from another perspective, of course. If no evidence about sameness of content is available in a given non-disagreement stand-off situation, then arguably the major argument for a non-indexical treatment of the relevant construction or expression is also not available ${ }^{18}$; in such a case the distinction between content context-sensitivity and circumstance contextsensitivity does not vindicate the rejection of an indexicalist analysis of conditional stand-offs and therefore of conditionals. A promising strategy for making an indexical treatment of (indicative) conditionals credible would therefore be to show that there is no evidence that, in a conditional stand-off situation, the speakers are affirming/denying the same conditional proposition ${ }^{19}$.

\footnotetext{
${ }^{18}$ Semantic Minimalists usually also make use of the foundational argument according to which semantic content must be clearly distinguished from pragmatic content. Thus Cappelen and Lepore claim that 'Semantic Minimalism ... is based on a correct understanding of the relationship between speech act content and semantic content: any view according to which it is a requirement on a semantic theory that it accounts for ... our basic ... intuitions about what speakers can use sentences to say, assert, state, etc., fails.... The resulting separation of speech act content and semantic content is necessary both in order to do semantics properly and in order to do speech act theory properly' (Cappelen and Lepore 2005: 15354). The same kind of argument can be found, for instance, in Salmon's 'Two Conceptions of Semantics' in Zoltán Gendler Szabó (ed.) Semantics versus Pragmatics (Oxford University Press, 2005), pp. 317-328. It is nevertheless somewhat question-begging, as it rejects the import of pragmatic elements to literal truthconditions on the basis of the idea that literal truth-conditions is the realm of semantics and that the boundary between semantics and pragmatics must not be blurred. Arguably, however, whether it should be blurred is part of what is under discussion when trying to make sense of cases of (non-standard) context-sensitivity.

${ }^{19}$ Of course, an even more promising strategy would be to effectively show that the conditional propositions being affirmed/denied are different and therefore that indexicalism about (at least indicative) conditionals is not only credible but true. I will not be pursuing this much more ambitious project here.
} 
Authors adopting minimalist views on context-sensitivity will, of course, be inclined to claim that such evidence is available. Cappelen and Lepore (2005), for instance, defend the idea that what could be called the 'fall-back' content of a sentence $S$ uttered in context $\mathrm{c}_{1}$ corresponds, by and large, to the minimal propositional content expressed by S - roughly, 'minimal' is supposed to designate a propositional content that does not incorporate any contextual information besides that resulting from the assignment of referents to (standard) indexicals (plus disambiguation and precisification of vague expressions). The 'fall-back' content of a given sentence $\mathrm{S}$ as uttered in context $\mathrm{c}_{1}$ is the content any competent speaker will be able to recover from hearing $\mathrm{S}$ in a different context $\mathrm{c}_{2}$ just by correctly assigning meanings to the words and phrases making up $\mathrm{S}$. The assumption here is that, although the speaker at $\mathrm{C}_{2}$ does not have access to the contextually relevant features of $\mathrm{c}_{1}$ enabling the original assertion of $\mathrm{S}$ to express an enriched meaning, viz. that resulting from the assignment of semantic values to the non-standard indexical items in S, she will still be able to correctly report the basic or minimal propositional content expressed by the original speaker at $\mathrm{c}_{1}$ (after indexicality, ambiguity, etc. have been dealt with). Building on the fact that this content is of a propositional nature, and also on the fact that it is common to all speech acts involving S, Cappelen and Lepore claim that such a 'fall-back' content is S's semantic content and determines S's (literal) truth-conditions, despite the fact that often it is also acknowledgely too unspecific to qualify as what most speakers would want to convey, or indeed 'say' ${ }^{20}$, in asserting S. This general conception of what counts as a sentence's minimal propositional content applies to most, if not all the instances of nonstandard context-sensitivity mentioned above. Gradable adjectives like 'tall', for instance, are a case in point. If a speaker utters 'Sara is tall' in $\mathrm{c}_{1}$, then what she literally said (the minimal, so to speak, propositional content she expressed) can be reported at $\mathrm{c}_{2}$ even though standards for tallness are different in $\mathrm{c}_{1}$ and $\mathrm{c}_{2}\left(\mathrm{c}_{1}\right.$ being, for instance, a context where super models are being assessed for

${ }^{20}$ As opposed to implicate, in particular. Much could be said about Cappelen and Lepore's notion of saying, and its connection with the distinction what is said/what is implicated (despite their own dismissal of the discussion as merely terminological), but space constraints prevent me form discussing it here. 
height thus forming the relevant comparison class, whereas in $\mathrm{C}_{2}$ the relevant comparison class is constituted, say, by seven-year olds). What is supposed to be at stake when one assigns a truthvalue to 'Sara is tall' is the minimal propositional content expressed by $\mathrm{S}$, one which is independent of any particular features concerning the comparison class relevant at each context. Hence, under this line of reasoning, intuitive judgements of falsity, respectively truth, for 'Sara is tall' in $\mathrm{c}_{1}$ and $\mathrm{c}_{2}$ (let us assume Sara is $1.65 \mathrm{~m}$ ) made on the basis of the comparison class being taken for granted at each context surely do not concern the semantic content proper of 'Sara is tall', nor do they do justice to the sentence's (minimal) truth-conditions. That this position seems to be committed to accepting an a-contextual property of tallness that people have or lack regardless of which criteria for tallness are in place in a given context does not bother Cappelen and Lepore. Furthermore, granting that the conception has less counterintuitive consequences in other cases of non-standard contextsensitivity, it vindicates the idea that minimal propositions have a role to play in a general account of linguistic communication. Since minimal propositions are the obvious candidate for the role of fall-back content, the minimalist stance on context-sensitivity is therefore argued to be vindicated.

This argument can be shown, nevertheless, to be a non sequitur. Interestingly enough, among the people that have qualms about it is a minimalist, Emma Borg ${ }^{21}$. Borg contends that the role of fallback content arguably played by minimal propositions does not show that speakers assert them and therefore that minimal propositions may be seen as determining a sentence's literal truthconditions. It is just, says Borg, that 'minimal propositions are ... the content any competent language user is guaranteed to be able to recover merely through exposure to the sentence uttered' and that 'indirect speech acts involving content sentences which share a literal meaning with the speaker's original utterance are typically judged correct' ${ }^{22}$, even if the content indirectly reported falls short, and is recognized as falling short, of the proposition actually

\footnotetext{
${ }^{21}$ Cf. Borg 2007. Borg herself endorses an even more radical version of minimalism (see for instance her Minimal Semantics, Oxford University Press, 2004).

${ }^{22}$ Borg 2007: 353-54.
} 
expressed by the original speaker. In other words, the role of fallback content played by minimal propositions in such cases does not ensure their role as the propositional content genuinely asserted by the utterer of the original sentence. Again, Borg's objection to Cappelen and Lepore's 'fall-back argument' is most clearly illustrated by the case of gradable adjectives: in the absence of information about the relevant comparison class, a speaker $\mathrm{X}$ may legitimately report John's assertion that Sara is tall by asserting 'John said that Sara is tall', without it being the case that she, or her audience, is aware of the comparison class the original speaker $\mathrm{Y}$ had in mind and therefore without it being the case that the content being reported is the proposition $\mathrm{Y}$ intended to put across. And, crucially, this is the case even if that proposition counts as the original utterance's literal content. In other words, even if (against Cappelen and Lepore) one accepts that $\mathrm{X}$ is not reporting the proposition literally expressed by the original utterance, one may still describe her report as correct. If this is so, then Cappelen and Lepore's fall-back argument fails to establish the role of the so-called minimal proposition as minimal asserted content.

Given that the intercontextual or fall-back content of an utterance need not be that utterance's minimal asserted content, the minimalist lacks a cogent argument for the claim that in a conditional stand-off the two intervening speakers are affirming/denying the same conditional proposition. If they were, that would mean that contextual idiosyncrasies pertaining to each speaker's communicative intentions would be irrelevant for the individuation of the conditional proposition being affirmed or denied; the literal truth-conditions of each conditional would be determined by what content they have after disambiguation and indexicality resolution, regardless of the particular criteria being used to substantiate its assertion (i.e. regardless of the particular criteria being used to assert or deny that the consequent is true if the antecedent is). In other words, those truth-conditions would be determined by what could be called the minimal propositional content of that conditional. But if we apply Borg's general point to the conditional case, there is no reason to describe the minimally asserted content of the conditional in a stand-off situation as that minimal proposition. Therefore, by Modus Tollens, the claim that in those cases the same conditional proposition is being asserted and denied is clearly weakened. 
By and large, then, given that conditional stand-offs are nodisagreement situations, the claim that the best account of them is along the lines of nonindexical contextualism is unsupported; the main motivation for a non-indexical treatment of the relevant perspectival idiosyncrasies is now missing. Indexicalism may well be the best option when trying to make sense of what is going on in a conditional stand-off.

Pedro Santos

Dept. de Línguas, Comunicação e Artes FCHS, Universidade do Algarve Campus de Gambelas 8005-139

Faro, Portugal psantos@ualg.pt

LanCog research group Centro de Filosofia, Universidade de Lisboa Faculdade de Letras, Alameda da Universidade 1600-214 Lisboa, Portugal

\section{References}

Borg. E. 2007. Minimalism versus Contextualism in Semantics. In Preyer, G. and Peter, G. (eds.) Context-Sensitivity and Semantic Minimalism. Oxford: Oxford University Press, pp. 339-359.

Cappelen, H. and Lepore, E. 2005. Insensitive Semantics. A Defense of Semantic Minimalism and Speech Act Pluralism. Oxford: Blackwell.

Edgington, D. 1995. On Conditionals. Mind 104: 235-329.

Edgington, D. 1997. Truth, Objectivity, Counterfactuals and Gibbard. Mind 106: 107-116.

Edgington, D. 2003. Conditionals, Truth and Objectivity. http://www.nyu.edu/gsas/dept/philo/courses/factual/papers/Edging ton.pdf

Gauker, C. 2005. Conditionals in Context. Cambridge, MA: MIT Press.

Gibbard, A. 1981. Two Recent Theories of Conditionals. In Harper, W. et al. (eds.) (1981) Ifs, D. Reidel, Dordrecht, 211-48.

Jackson, F. 1990. Classifying Conditionals. Analysis 50: 134- 47.

King, J. \& Stanley, J. 2005. Semantics, Pragmatics, and the Role of Semantic Content. In Zoltan Szabo (ed.) Semantics vs. Pragmatics. oxford: Oxford University Press, pp. 111-64.

Lowe, E.J. 1991. Jackson on Classifying Conditionals. Analysis 51: 126-30.

Lycan, W. 2001. Real Conditionals. Oxford: Clarendon Press. 
MacFarlane, J. (forthcoming). Indexical Contextualism. To appear in Synthese.

MacFarlane, J. 2007. Relativism and Disagreement. Philosophical Studies 132: 17-31.

Recanati, F. 2004. Literal Meaning. Cambridge: Cambridge University Press. 